\title{
CODESCENTE POUR LE NOYAU SAUVAGE ÉTALE
}

par

\author{
Hassan Asensouyis \& Jilali Assim
}

Résumé. - Soit $p$ un nombre premier impair et soit $M / F$ une $p$-extension galoisienne de corps de nombres, de groupe de Galois $G$. Nous étudions, pour tout entier $i \geq 2$, le noyau et le conoyau de l'homomorphisme naturel sur le noyau sauvage étale $N:\left(W K_{2 i-2}^{\text {ét }} M\right)_{G} \longrightarrow W K_{2 i-2}^{\text {ét }} F$, induit par la corestriction en cohomologie étale.

Abstract. - Let $p$ be an odd prime and let $M / F$ a Galois $p$-extension of number fields with Galois group $G$. We study, for $i \geq 2$, the kernel and cokernel of the natural map on the étale wild kernel $N:\left(W K_{2 i-2}^{\text {ét }} M\right)_{G} \longrightarrow W K_{2 i-2}^{\text {ét }} F$ induced by the corestriction in étale cohomology.

\section{Introduction}

Soit $M / F$ une extension galoisienne finie de corps de nombres, de groupe de Galois $G$. On se propose d'étudier, pour un nombre premier impair $p$, le noyau et le conoyau de l'homomorphisme

induit par la corestriction

$$
N:\left(W K_{2 i-2}^{\text {ét }} M\right)_{G} \longrightarrow W K_{2 i-2}^{\text {ét }} F
$$

$$
\text { cor : } H^{2}\left(M, \mathbb{Z}_{p}(i)\right) \longrightarrow H^{2}\left(F, \mathbb{Z}_{p}(i)\right)
$$

où, pour un corps $K$ quelconque, $j \geq 1, H^{j}\left(K, \mathbb{Z}_{p}(i)\right)$ est le $j$-ième groupe de cohomologie continue à coefficients dans le $i$-ème tordu de Tate $\mathbb{Z}_{p}(i)$. Rappelons que le noyau sauvage

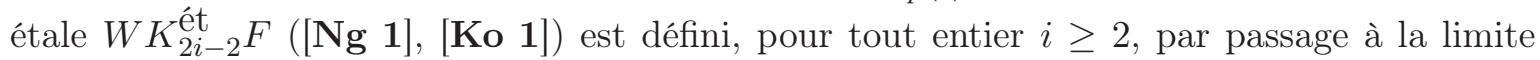
projective dans la suite exacte de Poitou-Tate, comme étant le noyau de l'homomorphisme de localisation

$$
H^{2}\left(F, \mathbb{Z}_{p}(i)\right) \longrightarrow \bigoplus_{v} H^{2}\left(F_{v}, \mathbb{Z}_{p}(i)\right)
$$

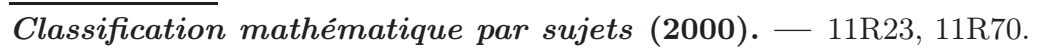

Mots clefs. - Noyau sauvage étale, codescente, $\lambda$-invariant.

Nous remercions le rapporteur pour de nombreuses remarques qui nous ont permis d'améliorer la rédaction. Le second auteur tient à remercier tous les organismes et personnes qui ont aidé à la réussite des premières Journées Arithmétiques de Meknès. 
où $v$ parcourt l'ensemble des places de $F$ et $F_{v}$ est le complété de $F$ en la place $v$.

L'homomorphisme $N$, qu'on peut voir comme l'analogue de l'application norme dans le cas du groupe de classes, a été étudié dans le cas d'une extension cyclique de degré $p$ par KolsterMovahhedi ([Ko-Mo]) et par Griffiths $([\mathbf{G r i}])$ dans sa thèse pour une extension cyclique de degré une puissance du nombre premier $p$. Dans cet article, nous étudions le cas d'une $p$-extension galoisienne quelconque. Nous déterminons explicitement le conoyau de $N$. En particulier le morphisme $N$ est surjectif sauf dans un cas exceptionnel pour lequel nous donnons une description arithmétique du conoyau. Pour calculer le noyau de $N$, on se heurte à des difficultés dûes notamment à la présence de certains groupes de cohomologie qui semblent être des obstructions à un principe de Hasse local-global "tordu". Ces difficultés, déjà observées dans le cas classique du groupe de classes (voir e.g $[\mathbf{N g} 3]$ ), ainsi que le défaut de surjectivité de $N$, disparaissent dans le cas où le degré $d$ de l'extension cyclotomique $F\left(\mu_{p}\right) / F$ ne divise pas l'entier $i-1$. Si $i \equiv 1(\bmod d)$, nous faisons l'hypothèse

$(\mathcal{H})$ : Il existe une p-place $v_{0}$ de $F$ qui ne se décompose pas ou une place $v_{0}$ totalement et modérément ramifiée dans l'extension $M / F$.

Si $F\left(\mu_{p}\right)$ contient le groupe des racines $p^{n}$-ièmes de l'unité, nous obtenons une formule des genres à la Chevalley pour une extension galoisienne de corps de nombres d'exposant $p^{n}$, généralisant celles de [Ko-Mo] et [Gri].

Enfin nous exploitons le cas simple des corps de nombres totalement réels et $i$ pair pour donner une démonstration de la formule de Riemann-Hurwitz $p$-adique (voir e.g. [I 2]). Le résultat obtenu donne une description explicite de la structure de $\mathbb{Z}_{p}[G]$-modules des "parties moins" du groupe de Galois de la pro-p-extension non-ramifiée abélienne maximale de $M\left(\mu_{p^{\infty}}\right)$, décomposant totalement toutes les places au-dessus de $p$.

\section{Préliminaires}

Pour tout corps de nombres $F$ d'anneau des entiers $O_{F}$ et tout nombre premier impair $p$, soit $S$ un ensemble de places de $F$ contenant l'ensemble $S_{p}$ des places au-dessus de $p$ et des places infinies. Notons $G_{S}=G_{S}(F)$ le groupe de Galois de l'extension algébrique $S$-ramifiée maximale de $F$.

Par passage à la limite projective dans la suite exacte de Poitou-Tate, nous avons la suite exacte

$$
H^{2}\left(G_{S}, \mathbb{Z}_{p}(i)\right) \rightarrow \bigoplus_{v \in S} H^{2}\left(F_{v}, \mathbb{Z}_{p}(i)\right) \rightarrow H^{0}\left(F, \mathbb{Q}_{p} / \mathbb{Z}_{p}(1-i)\right)^{*} \rightarrow 0
$$

où, pour un groupe $A, A^{*}$ est le dual de Pontryagin de $A$. Pour $i \neq 1$, le noyau de l'homomorphisme de localisation $H^{2}\left(G_{S}(F), \mathbb{Z}_{p}(i)\right) \rightarrow \bigoplus_{v \in S} H^{2}\left(F_{v}, \mathbb{Z}_{p}(i)\right)$ est indépendant de $S$ contenant $S_{p}([\mathbf{S c}])$. Pour $i=2$, les résultats de Tate $([\mathbf{T}])$ montrent que ce noyau est isomorphe à la partie $p$-primaire du noyau sauvage classique $([\mathbf{M}])$. Pour $i \geq 2$, il a été baptisé noyau sauvage étale par Kolster ([Ko 1]) et Nguyen Quang Do ([Ng 1]) et est noté $W K_{2 i-2}{ }_{\text {ét }}$, à cause notamment des liens entre les groupes de $K$-théorie algébrique et la cohomologie étale ([So], [D-F]). Les résultats de Soulé et Dwyer-Friedlander, et ceux de Borel sur la finitude des groupes de $K$-théorie algébrique $K_{2 i-2} O_{F}, i \geq 2$, entraînent en particulier que le noyau sauvage étale $W K_{2 i-2}^{\text {ét }} F$ est fini pour tout entier $i \geq 2$. Notons en passant que la finitude du 
noyau de l'homomorphisme de localisation ci-dessus est conjecturale pour $i \leq 0$ et que le cas $i=0$ correspond à la conjecture de Leopoldt ([Sc]). Si $i=1$, ce noyau n'est autre que la partie $p$-primaire du groupe des $S$-classes de $F$, i.e. le groupe de classes modulo les classes des diviseurs contenus dans $S$. Le noyau sauvage étale peut donc être considéré comme un analogue tordu du groupe de classes (tensorisé par $\mathbb{Z}_{p}$ ) et son étude recèle, comme celle du groupe de classes, des problèmes arithmétiques difficiles et intéressants (voir e.g [As], [Ko-Mo], [Ng 2], etc).

Soit maintenant $M / F$ une extension galoisienne $S$-ramifiée de corps de nombres, de groupe de Galois $G$. Nous avons alors deux applications de corestriction et restriction

$$
\text { cor : } H^{2}\left(G_{S}(M), \mathbb{Z}_{p}(i)\right) \rightarrow H^{2}\left(G_{S}(F), \mathbb{Z}_{p}(i)\right)
$$

et

$$
\text { res : } H^{2}\left(G_{S}(F), \mathbb{Z}_{p}(i)\right) \rightarrow H^{2}\left(G_{S}(M), \mathbb{Z}_{p}(i)\right)^{G}
$$

pour tout entier $i \geq 2$. Si $i=2$, ces applications correspondent respectivement aux homomorphismes transfert et extension pour le $K_{2}([\mathrm{M}])$. Il est bien connu que le noyau et le conoyau de l'homomorphisme res sont décrits à l'aide de la $G$-cohomologie de $H^{1}\left(G_{S}(M), \mathbb{Z}_{p}(i)\right)$ :

$$
\operatorname{ker}(\mathrm{res}) \cong H^{1}\left(G, H^{1}\left(G_{S}(M), \mathbb{Z}_{p}(i)\right)\right) \text { et } \operatorname{coker}(\text { res }) \cong H^{2}\left(G, H^{1}\left(G_{S}(M), \mathbb{Z}_{p}(i)\right)\right)
$$

([Ka], [CKPS], [Ko-Mo], etc), et que le morphisme de corestriction induit un isomorphisme, noté encore cor,

$$
H^{2}\left(G_{S}(M), \mathbb{Z}_{p}(i)\right)_{G} \stackrel{\sim}{\longrightarrow} H^{2}\left(G_{S}(F), \mathbb{Z}_{p}(i)\right)
$$

(voir e.g. [Ko 2]). Des résultats analogues sont valables dans le cas d'une extension de corps locaux.

Notons $\bigoplus_{v \in S} H^{2}\left(F_{v}, \mathbb{Z}_{p}(i)\right)$ le noyau de l'homomorphisme

$$
\bigoplus_{v \in S} H^{2}\left(F_{v}, \mathbb{Z}_{p}(i)\right) \rightarrow H^{0}\left(F, \mathbb{Q}_{p} / \mathbb{Z}_{p}(1-i)\right)^{*}
$$

Nous avons ainsi, pour tout entier $i \geq 2$, une suite exacte

$$
0 \rightarrow W K_{2 i-2}^{\text {ét }} F \rightarrow H^{2}\left(G_{S}, \mathbb{Z}_{p}(i)\right) \rightarrow \bigoplus_{v \in S}^{\sim} H^{2}\left(F_{v}, \mathbb{Z}_{p}(i)\right) \rightarrow 0
$$

Soit $\mu_{p}$ le groupe des racines $p$-ièmes de l'unité et soit $d:=\left[F\left(\mu_{p}\right): F\right]$. Il est clair que l'on a l'isomorphisme

$$
\bigoplus_{v \in S} H^{2}\left(F_{v}, \mathbb{Z}_{p}(i)\right) \cong \bigoplus_{v \in S} H^{2}\left(F_{v}, \mathbb{Z}_{p}(i)\right)
$$

exactement lorsque $d$ ne divise pas l'entier $i-1$. Notons

$$
N:\left(W K_{2 i-2}^{\text {ét }} M\right)_{G} \longrightarrow W K_{2 i-2}^{\text {ét }} F
$$

l'homomorphisme induit par la corestriction. Nous avons alors un diagramme commutatif : 


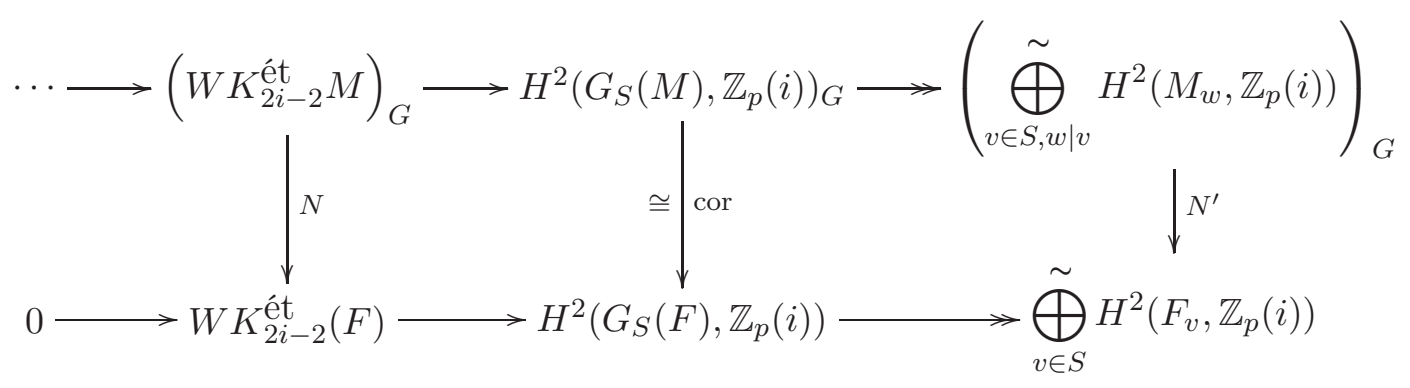

Ainsi $\operatorname{coker} N \simeq \operatorname{ker} N^{\prime}$ et

$$
\operatorname{ker} N \simeq \operatorname{coker}\left(H_{1}\left(G, H^{2}\left(G_{S}(M), \mathbb{Z}_{p}(i)\right)\right) \stackrel{\tilde{\alpha}}{\longrightarrow} H_{1}\left(G, \underset{w \mid v, v \in S}{\tilde{\bigoplus}} H^{2}\left(M_{w}, \mathbb{Z}_{p}(i)\right)\right)\right)
$$

\section{Détermination du conoyau}

Pour déterminer le conoyau de l'homomorphisme $N:\left(W K_{2 i-2}^{\text {ét }} M\right)_{G} \rightarrow W K_{2 i-2}^{\text {ét }} F$, considérons le diagramme commutatif

$$
\begin{aligned}
& \left(\bigoplus_{w \mid v, v \in S}^{\sim} H^{2}\left(M_{w}, \mathbb{Z}_{p}(i)\right)\right)_{G} \longrightarrow\left(\bigoplus_{w \mid v, v \in S} H^{2}\left(M_{w}, \mathbb{Z}_{p}(i)\right)\right)_{G} \longrightarrow\left(H^{0}\left(M, \mathbb{Q}_{p} / \mathbb{Z}_{p}(1-i)\right)^{*}\right)_{G} \\
& \downarrow^{\prime} \\
& \bigoplus_{v \in S} H^{2}\left(F_{v}, \mathbb{Z}_{p}(i)\right) \longleftrightarrow \bigoplus_{v \in S} H^{2}\left(F_{v}, \mathbb{Z}_{p}(i)\right) \\
& H^{0}\left(F, \mathbb{Q}_{p} / \mathbb{Z}_{p}(1-i)\right)^{*}
\end{aligned}
$$

dans lequel les deux flèches verticales de droite sont des isomorphismes. Si $i \not \equiv 1(\bmod d)$, on sait que les deux modules $\bigoplus_{w \mid v, v \in S}^{\sim} H^{2}\left(M_{w}, \mathbb{Z}_{p}(i)\right)$ et $\bigoplus_{w \mid v, v \in S} H^{2}\left(M_{w}, \mathbb{Z}_{p}(i)\right)$ coïncident et $N^{\prime}$ est donc un isomorphisme. En particulier $N$ est surjectif. On n'a donc à traiter que le cas $i \equiv 1(\bmod d)$.

Nous avons besoin de quelques notations supplémentaires :

$F_{\infty}$ : la $\mathbb{Z}_{p^{-}}$extension cyclotomique de $F$;

$\Gamma:=\operatorname{Gal}\left(F_{\infty} / F\right)$;

$L_{\infty}^{\prime}$ : la pro- $p$-extension abélienne non-ramifiée maximale de $F_{\infty}$, décomposant totalement toutes les places de $F_{\infty}$ au-dessus de $p$.

Proposition 2.1. - Soit $M / F$ une p-extension galoisienne de corps de nombres et supposons $i \equiv 1 \quad(\bmod d)$. Alors

$$
\operatorname{coker} N \cong \operatorname{Gal}\left(L_{\infty}^{\prime} \cap M_{\infty} / F_{\infty}\right)(i-1)_{\Gamma} .
$$


Démonstration. — Le diagramme commutatif ci-dessus montre que

$$
\operatorname{ker} N^{\prime} \cong \operatorname{coker}\left(H_{1}\left(G, \underset{w \mid v, v \in S}{\bigoplus} H^{2}\left(M_{w}, \mathbb{Z}_{p}(i)\right)\right) \rightarrow H_{1}\left(G, H^{0}\left(M, \mathbb{Q}_{p} / \mathbb{Z}_{p}(1-i)\right)^{*}\right)\right) \text {. }
$$

On sait que pour tout $v \in S, H^{2}\left(M_{w}, \mathbb{Z}_{p}(i)\right) \cong H^{0}\left(M_{w}, \mathbb{Q}_{p} / \mathbb{Z}_{p}(1-i)\right)^{*}$ (théorème de dualité locale). Utilisant le théorème de dualité pour la cohomologie des groupes finis, puis le lemme de Shapiro, il vient

$$
H_{1}\left(G, \bigoplus_{w \mid v, v \in S} H^{2}\left(M_{w}, \mathbb{Z}_{p}(i)\right)\right) \cong \bigoplus_{v \in S} H^{1}\left(G_{w}, H^{0}\left(M_{w}, \mathbb{Q}_{p} / \mathbb{Z}_{p}(1-i)\right)\right)^{*} .
$$

où, pour toute place finie $v$ de $S$, on a choisi une place $w$ au-dessus de $v$ et $G_{w}$ est le groupe de décomposition de $w$. Soit $H$ le groupe de Galois $\operatorname{Gal}\left(M_{\infty} / F_{\infty}\right)$ et pour une place finie $v$ quelconque, soient $H_{v}=\operatorname{Gal}\left(M_{w, \infty} / F_{v, \infty}\right), \Gamma_{w}=\operatorname{Gal}\left(M_{w, \infty} / M_{w}\right)$ et $\Gamma_{v}=\operatorname{Gal}\left(F_{v, \infty} / F_{v}\right)$. Par inflation-restriction, on a les suites exactes

$$
\begin{array}{r}
0 \longrightarrow H^{1}\left(G_{w}, H^{0}\left(\Gamma_{w}, \mathbb{Q}_{p} / \mathbb{Z}_{p}(1-i)\right)\right) \longrightarrow H^{1}\left(M_{w, \infty} / F_{v}, \mathbb{Q}_{p} / \mathbb{Z}_{p}(1-i)\right) \\
H^{1}\left(\Gamma_{w}, \mathbb{Q}_{p} / \mathbb{Z}_{p}(1-i)\right)^{G_{w}}=0
\end{array}
$$

et

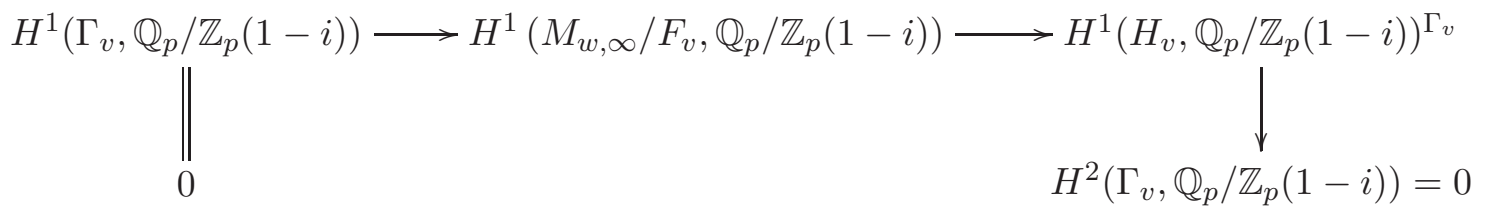

où la nullité des groupes $H^{j}\left(\Gamma_{w}, \mathbb{Q}_{p} / \mathbb{Z}_{p}(1-i)\right)$ et $H^{j}\left(\Gamma_{v}, \mathbb{Q}_{p} / \mathbb{Z}_{p}(1-i)\right), j=1,2$, découle du lemme de Tate (voir e.g.[Sc]). On en déduit que

$$
H^{1}\left(G_{w}, H^{0}\left(M_{w}, \mathbb{Q}_{p} / \mathbb{Z}_{p}(1-i)\right)\right) \cong H^{1}\left(H_{v}, \mathbb{Q}_{p} / \mathbb{Z}_{p}(1-i)\right)^{\Gamma_{v}} .
$$

Puisque $i \equiv 1 \quad(\bmod d), H_{v}$ opère trivialement sur $\mathbb{Q}_{p} / \mathbb{Z}_{p}(1-i)$, et donc

$$
H^{1}\left(G_{w}, H^{0}\left(M_{w}, \mathbb{Q}_{p} / \mathbb{Z}_{p}(1-i)\right)\right) \cong \operatorname{Hom}\left(H_{v}^{a b}(i-1)_{\Gamma_{v}}, \mathbb{Q}_{p} / \mathbb{Z}_{p}\right),
$$

ou encore

$$
H_{1}\left(G_{w}, H^{2}\left(M_{w}, \mathbb{Z}_{p}(i)\right)\right) \cong H_{v}^{a b}(i-1)_{\Gamma_{v}}
$$

De même :

$$
H_{1}\left(G, H^{0}\left(M, \mathbb{Q}_{p} / \mathbb{Z}_{p}(1-i)\right)\right) \cong H^{a b}(i-1)_{\Gamma}
$$

Par conséquent,

$$
\begin{aligned}
\operatorname{coker} N & \cong \operatorname{coker}\left(\bigoplus_{v \in S} H_{v}^{a b}(i-1)_{\Gamma_{v}} \longrightarrow H^{a b}(i-1)_{\Gamma}\right) \\
& =\operatorname{coker}\left(\bigoplus_{v \in S} H_{v}^{a b} \longrightarrow H^{a b}\right)(i-1)_{\Gamma}=\operatorname{Gal}\left(L_{\infty}^{\prime} \cap M_{\infty} / F_{\infty}\right)(i-1)_{\Gamma} .
\end{aligned}
$$


Corollaire 2.2. - Soit $M / F$ une p-extension galoisienne de corps de nombres et soit $L_{\infty}^{\prime}$ la pro-p-extension abélienne non-ramifiée maximale de $F_{\infty}$, décomposant totalement toutes les places au-dessus de p. Alors l'homomorphisme $N$ est surjectif exactement dans les cas suivants :
1. $i \not \equiv 1 \quad(\bmod d)$
2. $i \equiv 1 \quad(\bmod d)$ et $L_{\infty}^{\prime} \cap M_{\infty}=F_{\infty}$.

\section{Détermination du noyau}

3.1. Cas $i \equiv 1 \quad(\bmod d)$. — Rappelons que

$$
\operatorname{ker} N \cong \operatorname{coker}\left(H_{1}\left(G, H^{2}\left(G_{S}(M), \mathbb{Z}_{p}(i)\right)\right) \stackrel{\tilde{\alpha}}{\longrightarrow} H_{1}\left(G, \underset{w \mid v, v \in S}{\bigoplus} H^{2}\left(M_{w}, \mathbb{Z}_{p}(i)\right)\right)\right) \text {. }
$$

Malheureusement, le groupe $H_{1}(G, \overbrace{w \mid v, v \in S}^{\sim} H^{2}\left(M_{w}, \mathbb{Z}_{p}(i)\right))$ semble difficile à calculer. On a le diagramme :

$$
\begin{aligned}
& H_{2}\left(G, \bigoplus_{w \mid v, v \in S} H^{2}\left(M_{w}, \mathbb{Z}_{p}(i)\right)\right) \\
& \downarrow \\
& H_{2}\left(G, H^{0}\left(M, \mathbb{Q}_{p} / \mathbb{Z}_{p}(1-i)\right)^{*}\right) \\
& \downarrow \\
& H_{1}\left(G, H^{2}\left(G_{S}(M), \mathbb{Z}_{p}(i)\right)\right) \stackrel{\tilde{\alpha}}{\longrightarrow} H_{1}\left(G, \underset{w \mid v, v \in S}{\bigoplus_{\tilde{D}}} H^{2}\left(M_{w}, \mathbb{Z}_{p}(i)\right)\right) \\
& \cong H_{1}\left(G, H^{2}\left(G_{S}(M), \mathbb{Z}_{p}(i)\right)\right) \stackrel{\alpha}{\longrightarrow} H_{1}\left(G, \underset{w \mid v, v \in S}{\bigoplus} H^{2}\left(M_{w}, \mathbb{Z}_{p}(i)\right)\right) \\
& H_{1}\left(G, H^{0}\left(M, \mathbb{Q}_{p} / \mathbb{Z}_{p}(1-i)\right)^{*}\right)
\end{aligned}
$$

Supposons que l'hypothèse $(\mathcal{H})$ est vérifiée, c'est-à-dire qu'il existe une place $v_{0}$ de $S$ pour laquelle $G_{w_{0}} \cong G$ et

$$
H^{0}\left(M_{w_{0}}, \mathbb{Q}_{p} / \mathbb{Z}_{p}(1-i)\right) \cong H^{0}\left(M, \mathbb{Q}_{p} / \mathbb{Z}_{p}(1-i)\right),
$$

où $w_{0}$ est la place de $M$ au-dessus de $v_{0}$, et donc

$$
H_{j}\left(G, H^{0}\left(M_{w_{0}}, \mathbb{Q}_{p} / \mathbb{Z}_{p}(1-i)\right)\right) \cong H_{j}\left(G, H^{0}\left(M, \mathbb{Q}_{p} / \mathbb{Z}_{p}(1-i)\right)\right)
$$


pour $j=1,2$. Le diagramme ci-dessus et le théorème de dualité locale montrent alors que

$$
H_{j}\left(G, \bigoplus_{w \mid v, v \in S}^{\sim} H^{2}\left(M_{w}, \mathbb{Z}_{p}(i)\right)\right)=\bigoplus_{v \in S-\left\{v_{0}\right\}} H_{j}\left(G_{w}, H^{2}\left(M_{w}, \mathbb{Z}_{p}(i)\right)\right) .
$$

En résumé, sous l'hypothèse $(\mathcal{H})$, on a

$$
\operatorname{Ker} N \cong \operatorname{coker}\left(H_{1}\left(G, H^{2}\left(G_{S}(M), \mathbb{Z}_{p}(i)\right)\right) \longrightarrow \bigoplus_{v \in S-\left\{v_{0}\right\}} H_{1}\left(G_{w}, H^{2}\left(M_{w}, \mathbb{Z}_{p}(i)\right)\right)\right),
$$

où pour toute place $v \in S-\left\{v_{0}\right\}$, on a choisi une place $w$ de $M$ au-dessus de $v$ (lemme de Shapiro). On sait que ([CKPS], [Ko 2])

$$
H_{1}\left(G, H^{2}\left(G_{S}(M), \mathbb{Z}_{p}(i)\right)\right) \cong \widehat{H}^{0}\left(G, H^{1}\left(G_{S}(M), \mathbb{Z}_{p}(i)\right)\right)
$$

et pour tout $w \mid v, v \in S$

$$
H_{1}\left(G_{w}, H^{2}\left(M_{w}, \mathbb{Z}_{p}(i)\right)\right) \cong \widehat{H}^{0}\left(G_{w}, H^{1}\left(M_{w}, \mathbb{Z}_{p}(i)\right)\right)
$$

Par suite

$$
\operatorname{ker} N \cong \operatorname{coker}\left(\widehat{H}^{0}\left(G, H^{1}\left(G_{S}(M), \mathbb{Z}_{p}(i)\right)\right) \longrightarrow \bigoplus_{v \in S-\left\{v_{0}\right\}} \widehat{H}^{0}\left(G_{w}, H^{1}\left(M_{w}, \mathbb{Z}_{p}(i)\right)\right)\right)
$$

Puisque les groupes $H^{1}\left(F, \mathbb{Z}_{p}(i)\right)$ vérifient la descente galoisienne (voir e.g. [CKPS], [Ko 2]),

$$
\operatorname{Ker} N \cong \operatorname{coker}\left(\frac{H^{1}\left(F, \mathbb{Z}_{p}(i)\right)}{N_{G}\left(H^{1}\left(M, \mathbb{Z}_{p}(i)\right)\right)} \longrightarrow \bigoplus_{v \in S-\left\{v_{0}\right\}} \frac{H^{1}\left(F_{v}, \mathbb{Z}_{p}(i)\right)}{N_{G_{w}}\left(H^{1}\left(M_{w}, \mathbb{Z}_{p}(i)\right)\right)}\right) .
$$

Soit $m$ l'exposant de $G$, on a alors

$$
\operatorname{Ker} N \cong \operatorname{Coker}\left(\frac{H^{1}\left(F, \mathbb{Z}_{p}(i)\right) / m}{N_{G}\left(H^{1}\left(M, \mathbb{Z}_{p}(i)\right) / m\right)} \longrightarrow \bigoplus_{v \in S-\left\{v_{0}\right\}} \frac{H^{1}\left(F_{v}, \mathbb{Z}_{p}(i)\right) / m}{N_{G_{w}}\left(H^{1}\left(M_{w}, \mathbb{Z}_{p}(i)\right) / m\right)}\right)
$$

Pour simplifier, faisons $m=p^{n}$. Pour tout entier $i$, la suite exacte

$$
0 \rightarrow \mathbb{Z}_{p}(i) \stackrel{p^{n}}{\rightarrow} \mathbb{Z}_{p}(i) \rightarrow \mathbb{Z} / p^{n}(i) \rightarrow 0
$$

donne par cohomologie la suite exacte

$$
0 \rightarrow H^{1}\left(F_{v}, \mathbb{Z}_{p}(i)\right) / p^{n} \rightarrow H^{1}\left(F_{v}, \mathbb{Z} / p^{n}(i)\right) \rightarrow p^{n} H^{2}\left(F_{v}, \mathbb{Z}_{p}(i)\right) \rightarrow 0 .
$$

On suppose dans toute la suite de cette section que le corps $F\left(\mu_{p}\right)$ contient le groupe $\mu_{p^{n}}$ des racines $p^{n}$-ièmes de l'unité.

Proposition 3.1. - Soit $i \geq 2$. Pour tout $v \in S$, la surjection canonique

$$
\frac{H^{1}\left(F_{v}, \mathbb{Z}_{p}(i)\right) / p^{n}}{N_{G_{w}}\left(H^{1}\left(M_{w}, \mathbb{Z}_{p}(i)\right) / p^{n}\right)} \rightarrow \frac{H^{1}\left(F_{v}, \mathbb{Z}_{p}(i)\right) / p^{n}}{H^{1}\left(F_{v}, \mathbb{Z}_{p}(i)\right) / p^{n} \bigcap N_{G_{w}}\left(H^{1}\left(M_{w}, \mathbb{Z} / p^{n}(i)\right)\right)}
$$

est un isomorphisme. 
Démonstration. — Considérons le diagramme commutatif

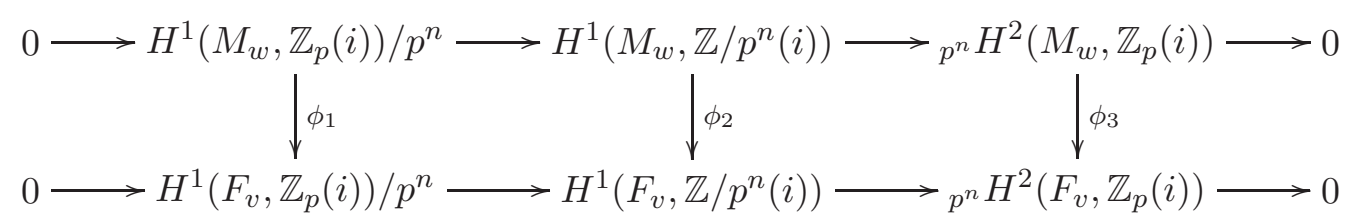

où $\phi_{1}$ et $\phi_{2}$ sont induites par la norme.

Pour prouver l'injection $\operatorname{coker}\left(\phi_{1}\right) \hookrightarrow \operatorname{coker}\left(\phi_{2}\right)$, il suffit de montrer que

$$
\left|\operatorname{coker}\left(\phi_{2}\right)\right|=\left|\operatorname{coker}\left(\phi_{1}\right)\right| \cdot\left|\operatorname{coker}\left(\phi_{3}\right)\right| \text {. }
$$

Puisque $\mu_{p^{n}} \subset F\left(\mu_{p}\right)$ et $i \equiv 1(\bmod d)$,

$$
H^{1}\left(M_{w}, \mathbb{Z} / p^{n}(i)\right) \cong H^{1}\left(M_{w}, \mu_{p^{n}}\right)(i-1) \cong M_{w}^{\bullet} / M_{w}^{\bullet p^{n}}(i-1)
$$

et

$$
H^{1}\left(F_{v}, \mathbb{Z} / p^{n}(i)\right) \cong F_{v}^{\bullet} / F_{v}^{\bullet p^{n}}(i-1) .
$$

Soit $M_{w}^{a b}$ l'extension abélienne maximale de $F_{v}$ contenue dans $M_{w}$. La théorie du corps de classes local montre alors que

$$
\operatorname{coker}\left(\phi_{2}\right) \simeq G_{w}^{a b} \simeq \operatorname{Gal}\left(M_{w}^{a b} / F_{v}\right) .
$$

Le calcul de l'ordre de coker $\left(\phi_{1}\right)$ découle de la preuve de la proposition 2.1 : puisque $H_{v}=$ $\operatorname{Gal}\left(M_{w, \infty} / F_{v, \infty}\right) \simeq \operatorname{Gal}\left(M_{w} / F_{v, \infty} \cap M_{w}\right)$ est tué par $p^{n}$ et $i \equiv 1(\bmod d)$, l'hypothèse $\mu_{p^{n}} \subseteq$ $F\left(\mu_{p}\right)$ entraîne que $H_{v}^{a b}(i-1)_{G_{w} / H_{v}} \cong\left(H_{v}^{a b}\right)_{G_{w} / H_{v}}(i-1)$. D'où

$$
\operatorname{coker}\left(\phi_{1}\right) \simeq \operatorname{Gal}\left(M_{w}^{a b} / F_{v, \infty} \cap M_{w}\right) .
$$

Pour calculer l'ordre de $\operatorname{coker}\left(\phi_{3}\right)$, il suffit de remarquer que $G_{w} / H_{v}$ agit trivialement sur $p^{n} H^{2}\left(M_{w}, \mathbb{Z}_{p}(i)\right) \cong \mathbb{Z} / p^{n}(i-1)$.

Le morphisme $\phi_{3}$ est donc l'élévation à la puissance $\left[F_{v, \infty} \cap M_{w}: F_{v}\right]$ et

$$
\left|\operatorname{coker}\left(\phi_{3}\right)\right|=\left|\operatorname{Gal}\left(F_{v, \infty} \cap M_{w} / F_{v}\right)\right| \text {. }
$$

Si $\mu_{p^{n}} \subseteq E:=F\left(\mu_{p}\right)$, on sait qu'il existe un sous-groupe $D_{F}^{(i, n)}$ de $E^{\bullet}$ tel que $H^{1}\left(F, \mathbb{Z}_{p}(i)\right) / p^{n} \cong$ $D_{F}^{(i, n)} / E^{\bullet p^{n}}(i-1)$ (voir e.g. [Gr], [As-Mo 1], [As-Mo 2], [V]). De plus, sous l'hypothèse $(\mathcal{H})$, le principe de Hasse est automatiquement vérifié. Nous obtenons ainsi une formule des genres dans le style de Chevalley généralisant celles de [Ko-Mo] (extensions cycliques de degré $p$ ) et [Gri] (extensions cycliques de degré une puissance de $p$ ) : 
Théorème 3.2. - Soit $M / F$ une extension galoisienne S-ramifiée de corps de nombres d'exposant $p^{n}$, de groupe de Galois $G$. Supposons $i \equiv 1(\bmod d)$. Alors, sous l'hypothèse $(\mathcal{H})$, l'homomorphisme

$$
N:\left(W K_{2 i-2}^{e ́ t} M\right)_{G} \longrightarrow W K_{2 i-2}^{e ́ t} F
$$

induit par la corestriction est surjectif. Si de plus $\mu_{p^{n}} \subseteq F\left(\mu_{p}\right)$,

$$
\frac{\left|\left(W K_{2 i-2}^{e ́ t} M\right)_{G}\right|}{\left|W K_{2 i-2}^{e ́ t} F\right|}=\frac{\prod_{v \in S}\left[M_{w, \infty}^{a b}: F_{v, \infty}\right]}{\left[M_{\infty}^{a b}: F_{\infty}\right]\left[D_{F}^{(i, n)}: D_{F}^{(i, n)} \cap N_{M / F} M^{\bullet}\right]}
$$

où $M_{\infty}^{a b}\left(\right.$ resp. $\left.M_{w, \infty}^{a b}\right)$ est l'extension abélienne maximale de $F$ (resp. $F_{v}$ ) contenue dans $M_{\infty}$ (resp. $\left.M_{w, \infty}\right)$.

Remarque 3.3. - Le groupe $D_{F}^{(i, n)}$, appelé noyau de Tate généralisé, est l'analogue du groupe des $p$-unités de $F$ (tensorisé par $\mathbb{Z}_{p}$ ). Comme dans le cas des $p$-unités, l'indice normique $\left[D_{F}^{(i, n)}: D_{F}^{(i, n)} \cap N_{M / F} M^{\bullet}\right]$ est difficile à calculer en général. Cependant, dans certains cas favorables, il est possible d'avoir plus de renseignements que dans le cas des $p$-unités. Considérons par exemple le cas où $M / F$ est une extension cyclique de degré $p$ et $F$ admet une seule place au-dessus de $p$. Soit $S$ l'ensemble des places au-dessus de $p$ et des places ramifiées dans l'extension $M / F$ et soit $U$ un ensemble primitif maximal contenu dans $S$ au sens de $[\mathbf{M o - N g}]$. Alors ([As-Mo 1])

$$
\left[D_{F}^{(i, n)}: D_{F}^{(i, n)} \cap N_{M / F} M^{\bullet}\right]=p^{u}
$$

où $u=\left|U \backslash S_{p}\right|$. Si $s=\left|S \backslash S_{p}\right|$ on a alors

$$
\frac{\left|\left(W K_{2 i-2}^{\text {ét }} M\right)_{G}\right|}{\left|W K_{2 i-2}^{\text {ét }} F\right|}=p^{s-u-1} .
$$

Le théorème de densité de Čebotarev assure l'existence d' une infinité d'ensembles primitifs. Dans [As-Mo 2], on donne d'autres exemples de calcul de l'indice normique ci-dessus en dehors du cas cyclique et de l'hypothèse $\mu_{p^{n}} \subseteq F$.

3.2. Cas $i \not \equiv 1 \quad(\bmod d)$. — On conserve les notations des sections précédentes. Si $i \not \equiv 1$ $(\bmod d)$, on sait que l'homomorphisme $N$ est surjectif (corollaire 2.2) et que

$$
\begin{aligned}
\operatorname{ker} N \cong \operatorname{coker}\left(H_{1}\left(G, H^{2}\left(G_{S}(M), \mathbb{Z}_{p}(i)\right)\right) \rightarrow \bigoplus_{v \in S} H_{1}\left(G_{w}, H^{2}\left(M_{w}, \mathbb{Z}_{p}(i)\right)\right)\right. \\
\cong \operatorname{coker}\left(\widehat{H}^{0}\left(G, H^{1}\left(G_{S}(M), \mathbb{Z}_{p}(i)\right)\right) \rightarrow \bigoplus_{v \in S} \widehat{H}^{0}\left(G_{w}, H^{1}\left(M_{w}, \mathbb{Z}_{p}(i)\right)\right)\right. \\
\cong \quad \operatorname{coker}\left(\frac{H^{1}\left(G_{S}(F), \mathbb{Z}_{p}(i)\right)}{N_{G} H^{1}\left(G_{S}(M), \mathbb{Z}_{p}(i)\right)} \rightarrow \bigoplus_{v \in S} \frac{H^{1}\left(F_{v}, \mathbb{Z}_{p}(i)\right)}{N_{G_{w}} H^{1}\left(M_{w}, \mathbb{Z}_{p}(i)\right)}\right)
\end{aligned}
$$

Concernant les groupes $H_{1}\left(G_{w}, H^{2}\left(M_{w}, \mathbb{Z}_{p}(i)\right)\right) \cong H^{1}\left(G_{w}, H^{0}\left(M_{w}, \mathbb{Q}_{p} / \mathbb{Z}_{p}(1-i)\right)\right)^{*}$, on voit que si $d_{v}:=\left[F_{v}\left(\mu_{p}\right): F_{v}\right]$ ne divise pas $i-1, H^{0}\left(M_{w}, \mathbb{Q}_{p} / \mathbb{Z}_{p}(1-i)\right)$ est trivial et si $d_{v}$ divise $i-1$, les mêmes calculs que dans le cas $i \equiv 1(\bmod d)$ montrent que $H_{1}\left(G_{w}, H^{2}\left(M_{w}, \mathbb{Z}_{p}(i)\right)\right) \cong$ $H_{v}^{a b}(i-1)_{\Gamma_{v}}$, où $\Gamma_{v}=\operatorname{Gal}\left(F_{v, \infty} / F_{v}\right)$ et $H_{v}=: \operatorname{Gal}\left(M_{w, \infty} / F_{v, \infty}\right)$. 
Soit

$$
S^{(i)}=\left\{v \in S ; d_{v} \mid(i-1)\right\} .
$$

Si $\mu_{p^{n}} \subset F\left(\mu_{p}\right)$ alors pour tout $v \in S^{(i)}$,

$$
\left|H_{1}\left(G_{w}, H^{2}\left(M_{w}, \mathbb{Z}_{p}(i)\right)\right)\right|=\left[M_{w, \infty}^{a b}: F_{v, \infty}\right],
$$

où, comme dans le théorème précédent, $M_{w, \infty}^{a b}$ est l'extension abélienne maximale de $F_{v}$ contenue dans $M_{w, \infty}$ De plus, d'après la la proposition 3.1,

$$
\frac{H^{1}\left(F_{v}, \mathbb{Z}_{p}(i)\right) / p^{n}}{N_{G_{w}}\left(H^{1}\left(M_{w}, \mathbb{Z}_{p}(i)\right) / p^{n}\right)} \stackrel{\sim}{\rightarrow} \frac{H^{1}\left(F_{v}, \mathbb{Z}_{p}(i)\right) / p^{n}}{H^{1}\left(F_{v}, \mathbb{Z}_{p}(i)\right) / p^{n} \bigcap N_{G_{w}}\left(H^{1}\left(M_{w}, \mathbb{Z} / p^{n}(i)\right)\right)}
$$

pour tout $v \in S^{(i)}$.

En résumé

Théorème 3.4. - Soit $M / F$ une extension galoisienne S-ramifiée de corps de nombres d'exposant $p^{n}$, de groupe de Galois $G$. On suppose que $i \not \equiv 1(\bmod d)$. Alors l'homomorphisme

$$
N:\left(W K_{2 i-2}^{e ́ t} M\right)_{G} \longrightarrow W K_{2 i-2}^{e ́ t} F
$$

induit par la corestriction est surjectif. Si de plus $\mu_{p^{n}} \subseteq F\left(\mu_{p}\right)$,

$$
|\operatorname{ker} N|=\frac{\prod_{v \in S^{(i)}}\left[M_{w, \infty}^{a b}: F_{v, \infty}\right]}{\left[D_{F}^{(i, n)}: D_{F}^{(i, n)} \bigcap \bigcap_{v \in S^{(i)}} N_{M_{w} / F_{v}} M_{w}^{\bullet}\right]}
$$

où, pour toute place $v \in S^{(i)}, M_{w, \infty}^{a b}$ est l'extension abélienne maximale de $F_{v}$ contenue dans $M_{w, \infty}$.

\section{Application}

Dans cette section, on applique la formule de co-descente obtenue dans le cas $i$ pair à une extension $M / F$ de corps de nombres totalement réels. Nous avons besoin de quelques notations supplémentaires.

Pour tout $\mathbb{Z}_{p}$-module $X$ sur lequel $\Delta$ opère et tout entier $j$,

$$
X^{[j]}=\left\{x \in X ; \forall \sigma \in \Delta, \sigma(x)=\omega^{j} . x\right\},
$$

où $\Delta$ est le groupe de $\operatorname{Galois} \operatorname{Gal}\left(F\left(\mu_{p}\right) / F\right) \simeq \operatorname{Gal}\left(F\left(\mu_{p^{\infty}}\right) / F_{\infty}\right)$ et $\omega$ est le caractère de Teichmüller.

Notons $X_{M}^{\prime}$ le groupe de Galois de la pro-p-extension non-ramifiée abélienne maximale de $M\left(\mu_{p} \infty\right)$, décomposant totalement toutes les places au-dessus de $p$. On sait que ([Sc])

$$
\lim _{\leftarrow}\left(W K_{2 i-2}^{\text {ét }} M_{n}\right) \cong X_{M}^{\prime[1-i]}(i-1) \text { et que } \lim _{\rightarrow}\left(W K_{2 i-2}^{\text {ét }} M_{n}\right) \cong \beta\left(X_{M}^{\prime[1-i]}(i-1)\right)
$$

où $\beta($.) désigne comme d'habitude le co-adjoint en théorie d'Iwasawa ([I 1], [W] chap. 15).

Notons aussi que puisque $M$ est totalement réel et que $i$ est pair,

$$
H^{2}\left(G_{S}\left(M_{n}\right), \mathbb{Z}_{p}(i)\right) \cong H^{1}\left(G_{S}\left(M_{n}\right), \mathbb{Q}_{p} / \mathbb{Z}_{p}(i)\right)
$$


pour tous les étages $M_{n}$ de la $\mathbb{Z}_{p}$-extension cyclotomique $M_{\infty}$ de $M([\mathbf{T}],[\mathbf{S c}])$. Par passage à la limite inductive dans la suite exacte (1), nous obtenons alors une suite exacte

$$
0 \rightarrow \beta\left(X_{M}^{\prime}[1-i](i-1)\right) \rightarrow H^{1}\left(G_{S}\left(M_{\infty}\right), \mathbb{Q}_{p} / \mathbb{Z}_{p}(i)\right) \rightarrow \underset{w \in S_{\infty}^{(i)}}{\bigoplus} \mathbb{Q}_{p} / \mathbb{Z}_{p}(i-1) \rightarrow 0
$$

où $S_{\infty}^{(i)}$ est l'ensemble des extensions à $M_{\infty}$ des places $v \in S$ telles que $\left[F_{v, \infty}\left(\mu_{p}\right): F_{v, \infty}\right]$ divise l'entier $i-1$. Remarquons que puisque $i$ est pair, $\left[F_{v, \infty}\left(\mu_{p}\right): F_{v, \infty}\right]$ divise $i-1$ exactement lorsque $v$ est totalement décomposée dans l'extension $F\left(\mu_{p}\right) / F$.

Supposons maintenant que $M / F$ est une extension cyclique de corps de nombres totalement réels, de degré $p$, de groupe de Galois $G$. Pour simplifier, on suppose que $M / F$ est linéairement disjointe de la $\mathbb{Z}_{p^{-}}$extension cyclotomique $F_{\infty} / F$; en particulier, $G \simeq \operatorname{Gal}\left(M_{n} / F_{n}\right)$ $\left(\simeq \operatorname{Gal}\left(M_{\infty} / F_{\infty}\right)\right)$ pour tous les étages $M_{n}, F_{n}$. Notons

$$
f_{\infty}: X_{F}^{\prime[1-i]} \rightarrow\left(X_{M}^{\prime}[1-i]\right)^{G}
$$

et

$$
N_{\infty}:\left(X_{M}^{\prime[1-i]}\right)_{G} \rightarrow X_{F}^{\prime[1-i]}
$$

les applications obtenues par passage à la limite projective sur les morphismes naturels $f_{n}: W K_{2 i-2}^{\text {ét }} F_{n} \rightarrow\left(W K_{2 i-2}^{\text {ét }} M_{n}\right)^{G}$ et $N_{n}:\left(W K_{2 i-2}^{\text {ét }} M_{n}\right)_{G} \rightarrow W K_{2 i-2}^{\text {ét }} F_{n}$ induits respectivement par la restriction et la corestriction. Puisque $i$ est pair et $M$ est totalement réel, le groupe $H^{1}\left(M, \mathbb{Z}_{p}(i)\right) \cong H^{0}\left(M, \mathbb{Q}_{p} / \mathbb{Z}_{p}(i)\right)$ est de torsion (voir e.g [Sc]). De la description cohomologique des noyaux et conoyaux des morphismes $f_{n}$, nous déduisons par passage à la limite projective que $f_{\infty}$ est injectif et que coker $f_{\infty}$ est fini. Supposons maintenant que l'invariant mu d'Iwasawa de $X_{M}^{\prime[1-i]}$ est nul. Il est bien connu que le module $X_{M}^{\prime[1-i]}$ est $\mathbb{Z}_{p}$-libre ([I 1], [W]). Il existe donc des entiers positifs $a_{1}, a_{p-1}, a_{p}$ tels que

$$
X_{M}^{\prime[1-i]} \cong \mathbb{Z}_{p}[G]^{a_{p}} \oplus I_{G}^{a_{p-1}} \oplus \mathbb{Z}_{p}^{a_{1}}
$$

où $I_{G}$ est l'idéal d'augmentation de $\mathbb{Z}_{p}[G]$ (théorème de Reiner). On se propose dans la suite de déterminer les quantités $a_{1}, a_{p-1}, a_{p}$.

Si $\lambda_{i}(F)$ désigne le $\mathbb{Z}_{p}$-rang du module $X_{F}^{\prime[1-i]}$, on voit que $a_{p}+a_{1}=\lambda_{i}(F)$ et que le quotient de Herbrand (en notation additive) $\chi\left(G, X_{M}^{\prime}[1-i]\right)=a_{1}-a_{p-1}$.

Soit $T$ l'ensemble des places $w \in S_{\infty}^{(i)}$ non-décomposées dans l'extension $M_{\infty} / F_{\infty}$ et soit $t$ le cardinal de $T$. La suite exacte (2) montre que

$$
\chi\left(G, \beta\left(X_{M}^{\prime[1-i]}\right)\right)=t+\chi\left(G, H^{1}\left(G_{S}\left(M_{\infty}\right), \mathbb{Q}_{p} / \mathbb{Z}_{p}(i)\right)\right) .
$$

Puisque $\alpha\left(X_{M}^{\prime[1-i]}\right):=\beta\left(X_{M}^{\prime[1-i]}\right)^{*}$ (l'adjoint en théorie d'Iwasawa) est pseudo-isomorphe à $X_{M}^{\prime[1-i]}$, il vient $\chi\left(G, X_{M}^{\prime[1-i]}\right)=-\chi\left(G, \beta\left(X_{M}^{\prime[1-i]}\right)\right)$. Par conséquent

$$
\chi\left(G, X_{M}^{\prime[1-i]}\right)=-t-\chi\left(G, H^{1}\left(G_{S}\left(M_{\infty}\right), \mathbb{Q}_{p} / \mathbb{Z}_{p}(i)\right)\right) .
$$

Les groupes $H^{q}\left(G_{S}\left(M_{\infty}\right), \mathbb{Q}_{p} / \mathbb{Z}_{p}(i)\right)$ sont nuls pour $q \geq 2$, la suite spectrale de HochschildSerre

$$
H^{p}\left(G, H^{q}\left(G_{S}\left(M_{\infty}\right), \mathbb{Q}_{p} / \mathbb{Z}_{p}(i)\right)\right) \Rightarrow H^{p+q}\left(G_{S}\left(F_{\infty}\right), \mathbb{Q}_{p} / \mathbb{Z}_{p}(i)\right)
$$

montre que $H^{1}\left(G, H^{1}\left(G_{S}\left(M_{\infty}\right), \mathbb{Q}_{p} / \mathbb{Z}_{p}(i)\right)\right) \simeq \mathbb{Z} / p \mathbb{Z}$ si $i \equiv 0(\bmod d), 0$ sinon et que 
$H^{2}\left(G, H^{1}\left(G_{S}\left(M_{\infty}\right), \mathbb{Q}_{p} / \mathbb{Z}_{p}(i)\right)\right)=0$. En particulier, $\chi\left(G, H^{1}\left(G_{S}\left(M_{\infty}\right), \mathbb{Q}_{p} / \mathbb{Z}_{p}(i)\right)\right)=-1$ si $i \equiv 0(\bmod d)$ et 0 sinon. D'où

$$
\chi\left(G, X_{M}^{\prime[1-i]}\right)=-t+1 \text { si } i \equiv 0 \quad(\bmod d) \text { et }-t \text { sinon. }
$$

Maintenant, on sait expliciter dans ce cas particulier ( $F$ totalement réel, $i$ pair) le noyau de l'homomorphisme $N_{\infty}$. On pourra ainsi déterminer la représentation galoisienne associée au module $X_{M}^{\prime[1-i]}$.

Pour $n$ assez grand, les places $v \in T$ ne se décomposent pas dans la tour cyclotomique $F_{\infty} / F_{n}$. Si $T=\emptyset$, il est clair que $N_{\infty}$ est injectif. Si $T \neq \emptyset, \operatorname{ker} N_{\infty} \simeq(\mathbb{Z} / p \mathbb{Z})^{t-1}$ si $d \mid i$ et $\operatorname{ker} N_{\infty} \simeq(\mathbb{Z} / p \mathbb{Z})^{t}$ sinon. D'un autre côté, il est clair que ker $N_{\infty} \simeq(\mathbb{Z} / p \mathbb{Z})^{a_{p-1}}$. Il en résulte que $a_{p-1}=t-1$ si $d \mid i$ et $T \neq \emptyset$ et $a_{p-1}=t$ sinon. Nous en déduisons que $a_{1}=1$ et $a_{p}=\lambda_{i}(F)-1$ si $T=\emptyset$ et $d \mid i$. Sinon, $a_{1}=0$ et $a_{p}=\lambda_{i}(F)$. En résumé

Théorème 4.1. - Soit $p$ un nombre premier impair et soit $M / F$ une extension cyclique de degré $p$, de groupe de Galois $G$, linéairement disjointe de la $\mathbb{Z}_{p}$-extension cyclotomique $F_{\infty} / F$. Supposons que l'invariant mu d'Iwasawa du module $X_{F}^{{ }^{[1-i]}}$ est nul et notons $\lambda_{i}(F)$ son invariant lambda. Soit $T$ l'ensemble des places de $F_{\infty}$ non-décomposées dans $M_{\infty} / F_{\infty}$ et totalement décomposées dans $F\left(\mu_{p} \infty\right) / F_{\infty}$ et soit $t$ son cardinal.

1. Si $T=\emptyset$ et $d \mid i$, nous avons un isomorphisme de $\mathbb{Z}_{p}[G]$-modules

$$
X_{M}^{\prime[1-i]} \cong \mathbb{Z}_{p}[G]^{\lambda_{i}(F)-1} \oplus \mathbb{Z}_{p} .
$$

2. $S i T=\emptyset$ et $d$ ne divise pas $i$

$$
X_{M}^{\prime[1-i]} \cong \mathbb{Z}_{p}[G]^{\lambda_{i}(F)} .
$$

3. Si T est non vide,

$$
X_{M}^{\prime}{ }^{[1-i]} \cong \mathbb{Z}_{p}[G]^{\lambda_{i}(F)} \oplus I_{G}^{t-\delta_{i}}
$$

où $\delta_{i}=1$ sid $\mid i$ et 0 sinon.

\section{Références}

[As] J. Assim, Analogues étales de la p-tour des corps de classes. J. Théor. Nombres Bordeaux 15 (2003), no. 3, 651-663.

[As-Mo 1] J. Assim and A. Movahhedi, Bounds for étale capitulation kernels. K-theory 33 (2004), 199-313.

[As-Mo 2] Assim, J. et A. Movahhedi, Norm index formulae and applications. À paraître dans J. of K-theory.

[CKPS] T. Chinburg, M. Kolster, G. Pappas, V. Snaith, Galois structure of K-groups of rings of integers. $K$-Theory 14 (1998), no. 4, 319-369.

[D-F] W. Dwyer, E. Friedlander, Algebraic and étale K-theory. Trans. Amer. Math. Soc. 247 (1985), 247-280.

[Gr] R. Greenberg, A note on $K_{2}$ and the theory of $\mathbb{Z}_{p}$-extensions. Amer. J. Math. 100 (1978), no. $6,1235-1245$.

[Gri] Ross A.W. Griffiths, A genus formula for étale Hilbert kernels in a cyclic p-power extension. PHD Thesis, McMaster University, 2005. 
[I 1] K. Iwasawa, On $\mathbb{Z}_{\ell}$-extensions of algebraic number fields. Ann. of Math. (2) 98 (1973) , 246-326.

[I 2] K. Iwasawa, Riemann-Hurwitz formula and p-adic Galois representations for number fields. Tôhoku Math. J. (2) 33 (1981), no. 2, 263-288.

[Ka] B. Kahn, Descente galoisienne et $K_{2}$ des corps de nombres. K-Theory 7 (1993), No.1, 55-100.

[Ko 1] M. Kolster, Remarks on étale K-theory and Leopoldt's conjecture. Séminaire de Théorie des Nombres, Paris, 1991-92, 37-62, Progr. Math., 116, Birkhäuser Boston, Boston, MA, 1993.

[Ko 2] M. Kolster, K-theory and arithmetic. Contemporary developments in algebraic K-theory. 191258 (electronic), ICTP Lect. Notes, XV, Abdus Salam Int. Cent. Theoret. Phys., Trieste, 2004.

[Ko-Mo] M. Kolster and A. Movahhedi, Galois co-descent for étale wild kernels and capitulation. Ann. Inst. Fourier 50 (2000), No.1, 35-65.

[M] M. Milnor, Introduction to algebraic K-theory,. Annals of Math. Studies 72, Princeton University Press, Princeton, 1971.

[Mo-Ng] A. Movahhedi et T. Nguyen Quang Do, Sur l'arithmétique des corps de nombres p-rationnels. Séminaire de Théorie des Nombres, Paris 1987-88, 155-200, Progr. Math., 81, Birkhäuser Boston, Boston, MA, 1990.

[Ng 1] T. Nguyen Quang Do, Analogues supérieurs du noyau sauvage Sém. Théor. Nombres Bordeaux (2) 4 (1992), no. 2, 263-271.

[Ng 2] T. Nguyen Quang Do, Théorie d'Iwasawa des noyaux sauvages étales d'un corps de nombres. Théorie des nombres, Années 1998/2001, 9 pp., Publ. Math. Besançon, 2002.

[Ng 3] T. Nguyen Quang Do, Quelques suites exactes en théorie des genres. Algèbre et théorie des nombres. Années 2003-2006, 103-115, Publ. Math. Besançon, 2006.

[Sc] P. Schneider, Über gewisse Galoiskohomologiegruppen. Math. Z. 168 (1979), 181-205.

[So] C. Soulé, K-théorie des anneaux d'entiers de corps de nombres et cohomologie étale. Inv. math 55 (1979), 251-295.

[T] J. Tate, Relations between $K_{2}$ and Galois cohomology. Invent. Math. 36 (1976), 257-274.

[V] D. Vauclair, Noyaux de Tate et capitulation. J. Number Theory 128 (2008), No. 3, 619-638.

[W] L. Washington, Introduction to cyclotomic fields, Springer, 1997.

12 décembre 2011

Hassan Asensouyis, Département de Mathématiques et Informatique, Université Moulay Ismail, B.P 11201 Zitoune, Meknès, Maroc • E-mail : rev.hassan@hotmail.com

Jilali Assim, Département de Mathématiques et Informatique, Université Moulay Ismail, B.P 11201 Zitoune, Meknès, Maroc • E-mail : assim@fs-umi.ac.ma 